NSF-ITP-99-128

hep-th/9910256

October 1999

\title{
Exact Renormalization Group and Loop Equation
}

\author{
Shinji Hirano \\ Institute for Theoretical Physics, \\ University of California, \\ Santa Barbara, CA 93106, USA
}

\begin{abstract}
We propose a gauge invariant formulation of the exact renormalization group equation for nonsupersymmetric pure $U(N)$ Yang-Mills theory, based on the construction by Tim Morris. In fact we show that our renormalization group equation amounts to a regularized version of the loop equation, thereby providing a direct relation between the exact renormalization group and the Schwinger-Dyson equations. We also discuss a possible implication of our formulation to the holographic correspondence of the bulk gravity and the boundary gauge theory.
\end{abstract}

\footnotetext{
${ }^{1}$ E-mail address: hirano@itp.ucsb.edu
} 


\section{Introduction}

The discovery of certain limits in string theory and M-theory can probably provide a reasoning for the dualities between quantum gravity and gauge theory. The celebrated examples are the light like limit [1] of M-theory, which at least literally justifies the Matrix conjecture [2], and the near horizon limits [3, 4] of D-branes and M-branes [5] that led us to recent excitement of the AdS/CFT duality [3, 6, 7]. Both the Matrix conjecture and the AdS/CFT duality intimately originates from the old $s$ - $t$ channel duality in open string loop amplitudes. It is, however, quite noteworthy that the discovery of the limits of [1, 3, 4 elaborated and complemented our intuitive but naive anticipation of a dual description of quantum gravity by a certain gauge theory, which may be based on the above-mentioned duality of open and closed strings.

The IR/UV relation, pointed out in [8, 9], itself is not surprising from the viewpoint of the $s$ - $t$ channel duality. But a rather conceptual payoff of this relation in the near horizon limit seems quite remarkable. In particular the identification of the radial coordinate $U$ in the near horizon geometry with the energy or the cutoff scale $\Lambda$ of the gauge theory clarifies the holographic nature of the AdS/CFT duality, in which it is quite plausibly assumed that the gauge theory contains only one degree of freedom per cell of the cutoff size.2 This postulate and the resultant holographic property actually fit in the basic idea of the Wilsonian renormalization group (Wilsonian RG) [10]. The assumption concerning degrees of freedom of the gauge theory is almost assured, if one refers to, for example, a lattice regularization. Once we regard the cutoff $\Lambda$ of the boundary gauge theory as the radial dimension in the bulk space, degrees of freedom of the bulk gravity may well be constituted solely from those of the boundary gauge theory, and the coarsening procedure or the RG transformation gives the dynamics in the interior space, which will likely be in general more complicated than that on the boundary. Thus we hope in this respect that the RG flow may correspond to the holographic mapping of the boundary data.

The motivation of this paper is to invent a formulation, on the gauge theory side, that might be a useful setting for discussing this hope. Since in the AdS/CFT duality gravitational modes in the bulk correspond to the gauge invariant operators on the boundary gauge theory, a gauge invariant formulation, in which the gauge redundancy is eliminated,

\footnotetext{
${ }^{2}$ There is in fact a subtlety in the relation between the radial coordinate $U$ and the cutoff scale $\Lambda$ [9]. The existence of two distinct relations is emphasized there. In both cases, however, there is a universal property that the cutoff scale $\Lambda$ increases with the radial scale $U$ up to dimensions 5 of the boundary gauge theory.

${ }^{3}$ The scale invariant theories, of course, do not have a nontrivial RG flow. We would, however, like to emphasize this point, suppose that the argument in [8] can be applied to more general nonconformal cases.
} 
may be much suited for discussing this sort of duality. In this regard we would like to respect a technique of collective field theory developed in [11], and apply it to a gauge invariant formulation of the exact $\mathrm{RG}$ equation.

There exist several papers [12, in which the authors discussed the RG interpretation of the AdS/CFT duality and some of them suggested the equivalence of the equation of motion in the AdS space (and its slight generalization) with the RG equation.f We hope that our formulation adds a new perspective along the line of their arguments.

\section{A Gauge Invariant Formulation of The Exact RG Equation}

Recently Tim Morris [14] proposed an exact RG equation for the Yang-Mills theory in a manifestly gauge invariant way, by constructing it in terms of a gauge invariant variable, a Wilson loop, just like the philosophy of the collective field method [11. His formulation is based on an observation inspired from a simple derivation [15] of the exact RG, that the exact $\mathrm{RG}$ equation is related to a particular field redefinition of the theory. For example, in scalar field theories in $D$ dimensions, the exact RG equation may be written in a form,

$$
\frac{\partial}{\partial \Lambda} e^{-S}=\int d^{D} \boldsymbol{x} \frac{\delta}{\delta \phi(\boldsymbol{x})}\left(\Psi[\phi(\boldsymbol{x})] e^{-S}\right)
$$

where $\Psi[\phi(\boldsymbol{x})]$ is induced from an infinitesimal change of the scalar field, $\phi(\boldsymbol{x}) \rightarrow \phi(\boldsymbol{x})+$ $\delta \Lambda \Psi[\phi(\boldsymbol{x})]$. The exact RG equation [15], which was employed to prove the renormalizability of the $\lambda \phi^{4}$ theory in four dimensions, is obtained by choosing the field redefinition as

$$
\Psi[\phi(\boldsymbol{x})]=\frac{1}{2} \int d^{D} \boldsymbol{y}\left[\dot{G}_{\Lambda}(\boldsymbol{x}-\boldsymbol{y}) \frac{\delta S}{\delta \phi(\boldsymbol{y})}-2\left(\dot{G}_{\Lambda} \cdot G_{\Lambda}^{-1}\right)(\boldsymbol{x}-\boldsymbol{y}) \phi(\boldsymbol{y})\right],
$$

where $G_{\Lambda}(\boldsymbol{x}-\boldsymbol{y})$ is the cutoff propagator of a massless scalar, defined by

$$
G_{\Lambda}(\boldsymbol{x}-\boldsymbol{y})=\int d^{D} \boldsymbol{p} \frac{1}{p^{2}} e^{i \boldsymbol{p} \cdot(\boldsymbol{x}-\boldsymbol{y})} K\left(p^{2} / \Lambda^{2}\right),
$$

in which $K\left(p^{2} / \Lambda^{2}\right)$ is a cutoff function that will take the value 1 for $p^{2}<\Lambda^{2}$ and vanish rapidly at infinity. Also $\dot{G}_{\Lambda}$ is the derivative of the propagator with respect to the cutoff $\Lambda$, i.e., $\dot{G}_{\Lambda}=\frac{\partial}{\partial \Lambda} G_{\Lambda}$.

In pure Yang-Mills theory in $D$ dimensions we may write the exact RG equation as

$$
\frac{\partial}{\partial \Lambda} e^{-S}=\operatorname{Tr} \int d^{D} \boldsymbol{x} \frac{\delta}{\delta A^{\mu}(\boldsymbol{x})}\left(\Psi^{\mu}\left[A^{\mu}(\boldsymbol{x})\right] e^{-S}\right) .
$$

\footnotetext{
${ }^{4}$ S.-J. Rey also bears an idea to develop this line of arguments [13].
} 
Here we introduced a standard convention, $A^{\mu}(\boldsymbol{x})=T^{a} A_{a}^{\mu}(\boldsymbol{x})$ and $\frac{\delta}{\delta A^{\mu}(\boldsymbol{x})}=T^{a} \frac{\delta}{\delta A_{a}^{\mu}(\boldsymbol{x})}$. A straightforward adaptation of the above regularization scheme (2), however, spoils the gauge symmetry. One way to avoid it is to look for some other form of the field redefinition (2) which maintains the gauge invariance. Indeed one such a choice was found in [14, in which a trick, the introduction of a pair of Wilson lines into the field redefinition, seemed to play an essential role. We make use of this trick, but we propose a somewhat similar but different formulation of the gauge invariant exact RG equation. In fact our formulation can be directly connected with the loop equation [16], as is rather different from the one proposed in [14].

Our choice of the field redefinition is

$$
\begin{aligned}
\Psi_{\mu}\left[A^{\mu}(\boldsymbol{x})\right]= & -\frac{1}{N \Lambda^{3}} \int d^{D} \boldsymbol{y} \int d^{D} \boldsymbol{p} e^{i \boldsymbol{p} \cdot(\boldsymbol{x}-\boldsymbol{y})}\left[K^{\prime}\left(p^{2} / \Lambda^{2}\right) \Phi[\Gamma \boldsymbol{x} \boldsymbol{y}] \frac{\delta S}{\delta A^{\mu}(\boldsymbol{y})} \Phi^{-1}[\Gamma \boldsymbol{x} \boldsymbol{y}]\right. \\
& +2 K^{\prime}\left(p^{2} / \Lambda^{2}\right) K^{-1}\left(p^{2} / \Lambda^{2}\right) \Phi[\Gamma \boldsymbol{x} \boldsymbol{y}] \frac{1}{2 g_{b}^{2}} D^{\nu} F_{\nu \mu}(\boldsymbol{y}) \Phi^{-1}[\Gamma \boldsymbol{x} \boldsymbol{y}] \\
& \left.-K^{\prime}\left(p^{2} / \Lambda^{2}\right) \frac{\delta}{\delta A_{a}^{\mu}(\boldsymbol{y})}\left(\Phi[\Gamma \boldsymbol{x} \boldsymbol{y}] T^{a} \Phi^{-1}[\Gamma \boldsymbol{x} \boldsymbol{y}]\right)\right] .
\end{aligned}
$$

This corresponds to the Fourier expansion of eq.(妀). Here $\Phi[\Gamma \boldsymbol{x} \boldsymbol{y}]$ is an advertised Wilson

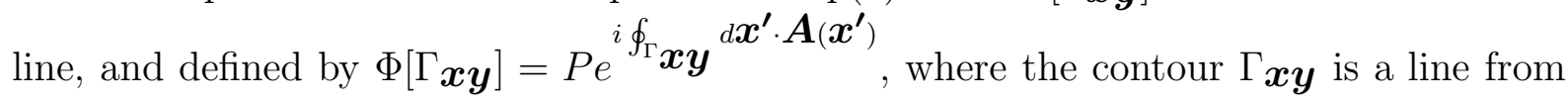
$\boldsymbol{x}$ to $\boldsymbol{y}$. Also $K^{\prime}(x)$ denotes the derivative of $K(x)$, i.e., $K^{\prime}(x)=\frac{d}{d x} K(x)$, and $g_{b}$ is the bare Yang-Mills coupling. In this form it is not clear whether the exact RG equation (4) is gauge invariant. We will, however, see below that it is indeed the case.

Now we can formally integrate the RG equation (4), and it takes the form

$$
e^{-S}=e_{D}^{\mathcal{H}\left[A, \frac{\delta}{\delta A} ; \Lambda\right]} e^{-S_{b}}
$$

with the bare action $S_{b}$ and

$$
\begin{aligned}
\mathcal{H}\left[A, \frac{\delta}{\delta A} ; \Lambda\right]= & \frac{1}{2 N} \int d^{D} \boldsymbol{x} \int d^{D} \boldsymbol{y} \int d^{D} \boldsymbol{p} \frac{1}{p^{2}} e^{i \boldsymbol{p} \cdot(\boldsymbol{x}-\boldsymbol{y})} \frac{\delta}{\delta A_{b}^{\mu}(\boldsymbol{x})}[ \\
& -K^{\prime}\left(p^{2} / \Lambda^{2}\right) \operatorname{Tr}\left(T^{b} \Phi[\Gamma \boldsymbol{x} \boldsymbol{y}] T^{a} \Phi^{-1}[\Gamma \boldsymbol{x} \boldsymbol{y}]\right) \frac{\delta}{\delta A_{a}^{\mu}(\boldsymbol{y})} \\
& +K^{\prime}\left(p^{2} / \Lambda^{2}\right) K^{-1}\left(p^{2} / \Lambda^{2}\right) \operatorname{Tr}\left(T^{b} \Phi[\Gamma \boldsymbol{x} \boldsymbol{y}] \frac{1}{g_{b}^{2}} D^{\nu} F_{\nu \mu}(\boldsymbol{y}) \Phi^{-1}[\Gamma \boldsymbol{x} \boldsymbol{y}]\right) \\
& \left.-K^{\prime}\left(p^{2} / \Lambda^{2}\right) \frac{\delta}{\delta A_{a}^{\mu}(\boldsymbol{y})} \operatorname{Tr}\left(T^{b} \Phi[\Gamma \boldsymbol{x} \boldsymbol{y}] T^{a} \Phi^{-1}[\Gamma \boldsymbol{x} \boldsymbol{y}]\right)\right]
\end{aligned}
$$

where the first functional derivative in the r.h.s. operates passing through the square 
brackets as well. Also $e_{D}^{\mathcal{H}\left[A, \frac{\delta}{\delta A} ; \Lambda\right]}$ denotes a Dyson series, 巳

$$
\sum_{n=0}^{\infty} \int_{0}^{\frac{1}{\Lambda^{2}}} d\left(1 / \Lambda_{n}^{2}\right) \int_{0}^{\frac{1}{\Lambda_{n}^{2}}} d\left(1 / \Lambda_{n-1}^{2}\right) \cdots \int_{0}^{\frac{1}{\Lambda_{1}^{2}}} d\left(1 / \Lambda_{0}^{2}\right) \mathcal{H}\left[A, \frac{\delta}{\delta A} ; \Lambda_{n}\right] \mathcal{H}\left[A, \frac{\delta}{\delta A} ; \Lambda_{n-1}\right] \cdots \mathcal{H}\left[A, \frac{\delta}{\delta A} ; \Lambda_{0}\right]
$$

Next let us consider the generating functional for Wilson loop correlators, as we are interested only in correlation functions of the gauge invariant operators. The generating functional is given by

$$
Z[J]=\int \mathcal{D} A^{\mu} \exp \left(-S+\sum_{C} J(C) W(C)\right)
$$

with the definition of the Wilson loop $W(C)=\frac{1}{N} \operatorname{Tr} P e^{i \oint_{C} d \boldsymbol{x} \cdot \boldsymbol{A}(\boldsymbol{x})}$. Using the integrated expression (6) of the exact RG equation and performing the integration by parts, we can rewrite it as

$$
Z[J]=\int \mathcal{D} A^{\mu} e^{-S_{b}}\left(e_{\widetilde{\mathcal{H}}\left[W, \frac{\delta}{\delta W} ; \Lambda\right]}^{\widetilde{\sum_{C} J(C) W(C)}}\right)
$$

where $e_{\widetilde{D}}^{\widetilde{\mathcal{H}}\left[W, \frac{\delta}{\delta W} ; \Lambda\right]}$ denotes a Dyson series in the reverse order to (8), i.e.,

$\sum_{n=0}^{\infty} \int_{0}^{\frac{1}{\Lambda^{2}}} d\left(1 / \Lambda_{n}^{2}\right) \int_{0}^{\frac{1}{\Lambda_{n}^{2}}} d\left(1 / \Lambda_{n-1}^{2}\right) \cdots \int_{0}^{\frac{1}{\Lambda_{1}^{2}}} d\left(1 / \Lambda_{0}^{2}\right) \widetilde{\mathcal{H}}\left[W, \frac{\delta}{\delta W} ; \Lambda_{0}\right] \widetilde{\mathcal{H}}\left[W, \frac{\delta}{\delta W} ; \Lambda_{1}\right] \cdots \widetilde{\mathcal{H}}\left[W, \frac{\delta}{\delta W} ; \Lambda_{n}\right]$

and $\widetilde{\mathcal{H}}\left[W, \frac{\delta}{\delta W} ; \Lambda\right]$ is written only in terms of Wilson loops. This shows the manifestation of gauge invariance in our formulation. We will relegate the detailed calculation of the operator $\widetilde{\mathcal{H}}\left[W, \frac{\delta}{\delta W} ; \Lambda\right]$ to the appendix. It finally comes up with a rather suggestive form which looks like the string field Hamiltonian,

$$
\begin{aligned}
& \widetilde{\mathcal{H}}\left[W, \frac{\delta}{\delta W} ; \Lambda\right]=\frac{1}{2} \int d^{D} \boldsymbol{p} \frac{1}{p^{2}}\left[K^{\prime}\left(p^{2} / \Lambda^{2}\right)\{\right. \\
& \frac{1}{N^{2}} \sum_{C, C^{\prime}} \int_{0}^{2 \pi} d s \int_{0}^{2 \pi} d s^{\prime}\left(\dot{\boldsymbol{x}}(s) \cdot \dot{\boldsymbol{x}}\left(s^{\prime}\right)\right) e^{i \boldsymbol{p} \cdot\left(\boldsymbol{x}\left(s^{\prime}\right)-\boldsymbol{x}(s)\right)} W\left(C_{M}\right) \frac{\delta}{\delta W\left(C^{\prime}\right)} \frac{\delta}{\delta W(C)} \\
& \left.+\sum_{C} \int_{0}^{2 \pi} d s \int_{0}^{2 \pi} d s^{\prime}\left(\dot{\boldsymbol{x}}(s) \cdot \dot{\boldsymbol{x}}\left(s^{\prime}\right)\right) e^{i \boldsymbol{p} \cdot\left(\boldsymbol{x}\left(s^{\prime}\right)-\boldsymbol{x}(s)\right)} W\left(C_{B}\right) W\left(C_{\bar{B}}\right) \frac{\delta}{\delta W(C)}\right\} \\
& \left.-\left.\frac{1}{g_{b}^{2} N} \int d^{D} \boldsymbol{y} K^{\prime}\left(p^{2} / \Lambda^{2}\right) K^{-1}\left(p^{2} / \Lambda^{2}\right) \sum_{C} \int_{0}^{2 \pi} d s e^{i \boldsymbol{p} \cdot(\boldsymbol{x}(s)-\boldsymbol{y})} \frac{\delta^{2} W(C)}{\delta x\left(s_{0}\right)^{2}}\right|_{\boldsymbol{x}\left(s_{0}\right)=\boldsymbol{y}} \frac{\delta}{\delta W(C)}\right] .
\end{aligned}
$$

\footnotetext{
${ }^{5}$ I would like to thank Marty Halpern for pointing out an error in the previous version.

${ }^{6}$ In the standard formulation of the exact RG, the source $J$ for a local operator is chosen in such a way that $J(p)$ is vanishing for higher momentum $p^{2}>\Lambda^{2}$ 115]. But the source $J(C)$ introduced here is the one for a non-local operator, and so it is unclear what choice is appropriate for the exact RG. We will not make any restrictions on the source at this stage, instead it will be constrained by the consistency of the exact RG equation, as we will see in the next section.
} 
As explained in the appendix, a loop $C_{M}$ denotes the merging of two loops $C$ and $C^{\prime}$, and is given by a product of four line contours as $C_{M}=C \boldsymbol{x}_{(s)} \boldsymbol{x}(s) \Gamma \boldsymbol{x}(s) \boldsymbol{x}\left(s^{\prime}\right) C \boldsymbol{x}\left(s^{\prime}\right) \boldsymbol{x}\left(s^{\prime}\right) \Gamma \boldsymbol{x}\left(s^{\prime}\right) \boldsymbol{x}(s)$. Also a pair of loops $C_{B}$ and $C_{\bar{B}}$ is broken up from a loop $C$, and they are expressed as $C_{B}=\Gamma \boldsymbol{x}_{\left(s^{\prime}\right)} \boldsymbol{x}(s) C_{\boldsymbol{x}(s)}{\boldsymbol{x}\left(s^{\prime}\right)}$ and $C_{\bar{B}}=C_{\boldsymbol{x}\left(s^{\prime}\right)} \boldsymbol{x}(s) \Gamma \boldsymbol{x}_{(s)} \boldsymbol{x}_{\left(s^{\prime}\right)}$. Here we distinguished a pair of lines $\Gamma_{\boldsymbol{x} y}$ introduced in the field redefinition (5) from the other lines $C_{\boldsymbol{x} y}$ which are parts of loops $C$ and $C^{\prime}$.

We will discuss later a possible implication of this result in the holographic correspondence of the bulk gravity and the boundary gauge theory.

\section{Loop Equation from The Exact RG Equation}

The Schwinger-Dyson equation is supposed to contain as much information as the exact RG equation, in a sense that, if we were to solve either of these two equations nonperturbatively, we could in principle obtain all the physical information of the quantum field theory. Thus we will be expected to have an intrinsic way to translate the Schwinger-Dyson equation into the exact RG equation, and vice versa, implicitly or explicitly. Actually we need to check if our proposed formulation really works, by, say, rederiving a known result obtained from a reliable formulation. In this respect it turns out interestingly enough that our exact RG equation amounts to a regularized version of the loop equation of [16], while this result is not so surprising and in fact rather reasonable, as we just mentioned. To see it, let us note that eq.(10) can be further rewritten into the form

$$
Z[J]=e_{D}^{\widetilde{\mathcal{H}}\left[\frac{\delta}{\delta J}, J ; \Lambda\right]} Z_{b}[J]
$$

where $Z_{b}[J]$ is the generating functional of the bare form, i.e.,

$$
Z_{b}[J]=\int \mathcal{D} A^{\mu} \exp \left(-S_{b}+\sum_{C} J(C) W(C)\right),
$$

and $e_{D}^{\widetilde{\mathcal{H}}\left[\frac{\delta}{\delta J}, J ; \Lambda\right]}$ is a Dyson series in the same order as (8). Also in the operator $\widetilde{\mathcal{H}}\left[\frac{\delta}{\delta J}, J ; \Lambda\right]$ the derivatives $\frac{\delta}{\delta J}$ 's are ordered on the right side of the sources $J$ 's.

Now the exact RG equation implies

$$
\frac{d}{d \Lambda} Z[J]=0
$$

This is equivalent to

$$
0=\int d^{D} \boldsymbol{p} \frac{1}{p^{2}}\left(\frac{\partial}{\partial \Lambda} K\left(p^{2} / \Lambda^{2}\right)\right) K^{-1}\left(p^{2} / \Lambda\right) \sum_{C} J(C) \int_{0}^{2 \pi} d s[
$$




$$
\begin{aligned}
& \frac{1}{N^{2}} \sum_{C^{\prime}} J\left(C^{\prime}\right) \int_{0}^{2 \pi} d s^{\prime}\left(\dot{\boldsymbol{x}}(s) \cdot \dot{\boldsymbol{x}}\left(s^{\prime}\right)\right) K\left(p^{2} / \Lambda^{2}\right) e^{i \boldsymbol{p} \cdot\left(\boldsymbol{x}\left(s^{\prime}\right)-\boldsymbol{x}(s)\right)} \frac{\delta Z[J]}{\delta J\left(C_{M}\right)} \\
& +\int_{0}^{2 \pi} d s^{\prime}\left(\dot{\boldsymbol{x}}(s) \cdot \dot{\boldsymbol{x}}\left(s^{\prime}\right)\right) K\left(p^{2} / \Lambda^{2}\right) e^{i \boldsymbol{p} \cdot\left(\boldsymbol{x}\left(s^{\prime}\right)-\boldsymbol{x}(s)\right)} \frac{\delta^{2} Z[J]}{\delta J\left(C_{B}\right) \delta J\left(C_{\bar{B}}\right)} \\
& \left.-\left.\frac{1}{g_{b}^{2} N} \int d^{D} \boldsymbol{y} e^{i \boldsymbol{p} \cdot(\boldsymbol{x}(s)-\boldsymbol{y})} \frac{\delta^{2}}{\delta x\left(s_{0}\right)^{2}} \frac{\delta Z[J]}{\delta J(C)}\right|_{\boldsymbol{x}\left(s_{0}\right)=\boldsymbol{y}}\right]
\end{aligned}
$$

Therefore we may recognize that the quantity in the square bracket is vanishing, and then integrating over the momentum $\boldsymbol{p}$, we come up to the loop equation with a regularization,

$$
\begin{aligned}
& \frac{1}{g_{b}^{2} N} \frac{\delta^{2}}{\delta x(s)^{2}} \frac{\delta Z[J]}{\delta J(C)} \\
& \quad=\frac{1}{N^{2}} \sum_{C^{\prime}} J\left(C^{\prime}\right) \int_{0}^{2 \pi} d s^{\prime}\left(\dot{\boldsymbol{x}}(s) \cdot \dot{\boldsymbol{x}}\left(s^{\prime}\right)\right) \int d^{D} \boldsymbol{p} K\left(p^{2} / \Lambda^{2}\right) e^{i \boldsymbol{p} \cdot\left(\boldsymbol{x}\left(s^{\prime}\right)-\boldsymbol{x}(s)\right)} \frac{\delta Z[J]}{\delta J\left(C_{M}\right)} \\
& \quad+\int_{0}^{2 \pi} d s^{\prime}\left(\dot{\boldsymbol{x}}(s) \cdot \dot{\boldsymbol{x}}\left(s^{\prime}\right)\right) \int d^{D} \boldsymbol{p} K\left(p^{2} / \Lambda^{2}\right) e^{i \boldsymbol{p} \cdot\left(\boldsymbol{x}\left(s^{\prime}\right)-\boldsymbol{x}(s)\right)} \frac{\delta^{2} Z[J]}{\delta J\left(C_{B}\right) \delta J\left(C_{\bar{B}}\right)} .
\end{aligned}
$$

Note that interestingly the cutoff function $K\left(p^{2} / \Lambda^{2}\right)$ enters in an expected way. In fact $\int d^{D} \boldsymbol{p} K\left(p^{2} / \Lambda^{2}\right) e^{i \boldsymbol{p} \cdot\left(\boldsymbol{x}\left(s^{\prime}\right)-\boldsymbol{x}(s)\right)}$ can be thought of as a smeared $\delta$-function, which is necessary to regularize the loop equation. Also loops are ordinarily closed by this $\delta$-function, and so the smearing of the $\delta$-function might undergo a potential breakdown of the gauge symmetry. It is, however, obvious from our explicit computation that loops are closed in spite of the smearing of the $\delta$-function in our formulation, due to a pair of Wilson lines introduced in the field redefinition (5). [1]

\section{Discussion}

As emphasized in the introduction, the cutoff scale $\Lambda$ of the gauge theory can be regarded as the radial scale $U$ in the AdS space, or more generally in the near horizon geometries of $D p$-branes 9.8 This simple but significant observation led us to contemplate the RG interpretation of the bulk/boundary duality, and pursue a gauge invariant formulation of the exact RG equation for the Yang-Mills theory. In particular to discuss the AdS/CFT duality, we apparently need to consider the supersymmetric extension of our formulation. For this purpose the analysis in [19, 20] and a supersymmetric Wilson loop in [21] are certainly of importance. We would, however, like to discuss a possibility implied by our

\footnotetext{
${ }^{7}$ This regularization seems similar to the one proposed in [18]. In a review article, they summarized extensive applications of their regularization scheme, based on the stochastic quantization, to a variety of quantum field theories.

${ }^{8}$ Again we should be careful with the distinction between holographic and D-brane probes. See a footnote in the introduction.
} 
formulation in rather wider context of the holographic correspondence between the bulk gravity and the boundary gauge theory.

According to the wisdom of the AdS/CFT duality, a disturbance on the boundary will be responded by the bulk gravity as a gravitational fluctuation, in such a way that

$$
\left\langle\exp \left(\int_{\partial M} d^{D} \boldsymbol{x} \phi_{0}(\boldsymbol{x}) \mathcal{O}(\boldsymbol{x})\right)\right\rangle=\exp \left(-S_{\text {grav }}[\phi(\boldsymbol{x}, U)]\right),
$$

where $\partial M$ is the boundary of the bulk space $M$, and $\mathcal{O}(\boldsymbol{x})$ is a local operator on the boundary field theory. Also $\phi(\boldsymbol{x}, U)$ is a gravitational mode on the bulk space, and it becomes $\phi_{0}(\boldsymbol{x})$ at the boundary. Furthermore in the gravity action $S_{\text {grav }}$ the gravitational mode $\phi(\boldsymbol{x}, U)$ is subject to the equation of motion, so that the r.h.s. depends only on the boundary value $\phi(\boldsymbol{x}, \infty)=\phi_{0}(\boldsymbol{x})$ of a gravitational mode.

If we insist on a stronger conjecture [3] on this duality that the string theory in the bulk is dual to the finite $N$ boundary gauge theory, we may consider the correlation functions of Wilson loops $W(C)$ instead of local operators $\mathcal{O}(\boldsymbol{x})$. Then the gravity action might be replaced by the string field action. From this viewpoint we would like to recall our result (13) on the generating functional of Wilson loop correlators,

$$
\left\langle\exp \left(\sum_{C} J(C) W(C)\right)\right\rangle=Z[J]=e_{D}^{\widetilde{\mathcal{H}}[\delta, J j, \Lambda]} Z_{b}[J] .
$$

Now let us put the RG interpretation of the bulk/boundary duality into this argument. The bulk physics at some scale $U$ may be given by the boundary gauge theory in which degrees of freedom at higher momentum modes than the cutoff scale $\Lambda$ are integrated out. In this regard $Z_{b}[J]$ denotes the unintegrated form of the generating functional, and so it corresponds to the bulk physics at the IR limit or the boundary. Then the operator $\widetilde{\mathcal{H}}\left[\frac{\delta}{\delta J}, J ; \Lambda\right]$ gives the RG flow from UV to IR of the boundary gauge theory, that corresponds to a mapping from the boundary to the interior of the bulk gravity. In this sense we would like to regard the RG flow operator $\widetilde{\mathcal{H}}\left[\frac{\delta}{\delta J}, J ; \Lambda\right]$ as the holographic mapping of the boundary data. Moreover as we discussed in the last section, the RG equation implies $\frac{d}{d \Lambda} Z[J]=0$, which is tantamount to a regularized version of the loop equation. Thus we might interpret the regularized loop equation as the string field equation of motion in the bulk space. Remember also that the RG flow operator $\widetilde{\mathcal{H}}\left[\frac{\delta}{\delta J}, J ; \Lambda\right]$ has a form of the string field Hamiltonian which consists of the terms that describe the joining and the splitting of strings and the string propagation. These facts fit at least literally in a stronger conjecture on the bulk/boundary duality.

Apart from the RG interpretation of the bulk/boundary duality, we would like to mention a similarity of our formulation with the stochastic quantization of the YangMills theory. In fact the authors in 22] proposed an interpretation of the Fokker-Planck 
Hamiltonian of the Yang-Mills theory as the string field Hamiltonian, initially as the one in the temporal gauge, just as discussed in the non-critical string theory, and later speculated an alternative interpretation that the fictitious time $\tau$ of the stochastic quantization can be thought of as the radial coordinate $U$ of the AdS space. If Our formulation is close to their latter speculation. This similarity originates from the fact that the exact RG and the Fokker-Planck equations are quite similar diffusion equations with the cutoff $\Lambda$ and the fictitious time $\tau$ respectively as the time. It, however, seems hard to give a physical meaning to the finite value of the fictitious time $\tau$ in the stochastic quantization .

\section{Acknowledgment}

We would like to thank Hajime Aoki, Aki Hashimoto, Marty Halpern, Kentaro Hori, Mitsuhiro Kato, Joe Polchinski, Soo-Jong Rey and Rikard von Unge for useful discussions and comments. This work was supported in part by the Japan Society for the Promotion of Science and by the National Science Foundation under Grant No. PHY94-07194.

\section{Appendix}

The computation of $\widetilde{\mathcal{H}}\left[W, \frac{\delta}{\delta W} ; \Lambda\right]$ can be done by mixture of the technique of the collective field method in [11] and that of [17] presented in a derivation of the loop equation. In terms of the gauge fields $A^{\mu}(\boldsymbol{x})$, it is expressed as

$$
\begin{aligned}
\widetilde{\mathcal{H}}\left[W, \frac{\delta}{\delta W} ; \Lambda\right]= & \frac{1}{2 N} \int d^{D} \boldsymbol{x} \int d^{D} \boldsymbol{y} \int d^{D} \boldsymbol{p} \frac{1}{p^{2}} e^{i} \boldsymbol{p} \cdot(\boldsymbol{x}-\boldsymbol{y})[ \\
& -K^{\prime}\left(p^{2} / \Lambda^{2}\right) \operatorname{Tr}\left(T^{b} \Phi[\Gamma \boldsymbol{x} \boldsymbol{y}] T^{a} \Phi^{-1}[\Gamma \boldsymbol{x} \boldsymbol{y}]\right) \frac{\delta^{2}}{\delta A_{a}^{\mu}(\boldsymbol{y}) \delta A_{b}^{\mu}(\boldsymbol{x})} \\
& \left.-K^{\prime}\left(p^{2} / \Lambda^{2}\right) K^{-1}\left(p^{2} / \Lambda^{2}\right) \operatorname{Tr}\left(T^{b} \Phi[\Gamma \boldsymbol{x} \boldsymbol{y}] \frac{1}{g_{b}^{2}} D^{\nu} F_{\nu \mu}(\boldsymbol{y}) \Phi^{-1}[\Gamma \boldsymbol{x} \boldsymbol{y}]\right) \frac{\delta}{\delta A_{b}^{\mu}(\boldsymbol{x})}\right] .
\end{aligned}
$$

The second derivative term consists of two pieces, the joining and the splitting of strings, due to the chain rule, as in (the first paper of) [11]. The one that describes the joining of strings is

$$
\sum_{C, C^{\prime}} \operatorname{Tr}\left(T^{b} \Phi[\Gamma \boldsymbol{x} \boldsymbol{y}] T^{a} \Phi^{-1}[\Gamma \boldsymbol{x} \boldsymbol{y}]\right) \frac{\delta W(C)}{\delta A_{a}^{\mu}(\boldsymbol{y})} \frac{\delta W\left(C^{\prime}\right)}{\delta A_{b}^{\mu}(\boldsymbol{x})} \frac{\delta}{\delta W\left(C^{\prime}\right)} \frac{\delta}{\delta W(C)}
$$

\footnotetext{
${ }^{9}$ Actually the string field theory in the temporal gauge of non-critical strings was first proposed in [23], and subsequently it was reconstructed in 24] as a collective field theory of stochastic quantization of matrix models in the double scaling limit. Also the intrinsic equivalence of the Fokker-Planck Hamiltonian and the loop operator was pointed out in [25]. The authors in 22] patched those ideas together, and added a new interpretation in the context of the Polyakov's non-critical strings [26] and also of the AdS/CFT duality.
} 
Actually the joining property can be easily understood from an explicit calculation.

$$
\begin{aligned}
& N^{2} \operatorname{Tr}\left(T^{b} \Phi[\Gamma \boldsymbol{x} \boldsymbol{y}] T^{a} \Phi^{-1}[\Gamma \boldsymbol{x y}]\right) \frac{\delta W(C)}{\delta A_{a}^{\mu}(\boldsymbol{y})} \frac{\delta W\left(C^{\prime}\right)}{\delta A_{b}^{\mu}(\boldsymbol{x})} \\
& =-\int_{0}^{2 \pi} d s \int_{0}^{2 \pi} d s^{\prime}\left(\dot{\boldsymbol{x}}(s) \cdot \dot{\boldsymbol{x}}\left(s^{\prime}\right)\right) \delta^{D}(\boldsymbol{x}(s)-\boldsymbol{y}) \delta^{D}\left(\boldsymbol{x}\left(s^{\prime}\right)-\boldsymbol{x}\right) \\
& \times \operatorname{Tr}\left[\left(P e^{i \int_{0}^{s} d s^{\prime \prime} \dot{\boldsymbol{x}}\left(s^{\prime \prime}\right) \cdot \boldsymbol{A}\left(\boldsymbol{x}\left(s^{\prime \prime}\right)\right)}\right) \Phi^{-1}[\Gamma \boldsymbol{x} \boldsymbol{y}]\left(P e^{i \int_{s^{\prime}}^{2 \pi} d s^{\prime \prime} \dot{\boldsymbol{x}}\left(s^{\prime \prime}\right) \cdot \boldsymbol{A}\left(\boldsymbol{x}\left(s^{\prime \prime}\right)\right)}\right)\right. \\
& \left.\times \quad\left(P e^{i \int_{0}^{s^{\prime}} d s^{\prime \prime} \dot{\boldsymbol{x}}\left(s^{\prime \prime}\right) \cdot \boldsymbol{A}\left(\boldsymbol{x}\left(s^{\prime \prime}\right)\right)}\right) \Phi[\Gamma \boldsymbol{x} \boldsymbol{y}]\left(P e^{i \int_{s}^{2 \pi} d s^{\prime \prime} \dot{\boldsymbol{x}}\left(s^{\prime \prime}\right) \cdot \boldsymbol{A}\left(\boldsymbol{x}\left(s^{\prime \prime}\right)\right)}\right)\right] \\
& =-N \int_{0}^{2 \pi} d s \int_{0}^{2 \pi} d s^{\prime}\left(\dot{\boldsymbol{x}}(s) \cdot \dot{\boldsymbol{x}}\left(s^{\prime}\right)\right) \delta^{D}(\boldsymbol{x}(s)-\boldsymbol{y}) \delta^{D}\left(\boldsymbol{x}\left(s^{\prime}\right)-\boldsymbol{x}\right) W\left(C_{M}\right),
\end{aligned}
$$

where a loop $C_{M}$ denotes the merging of two loops $C$ and $C^{\prime}$, and it is composed of four lines, i.e., $C_{M}=C_{\boldsymbol{x}(s)} \boldsymbol{x}_{(s)} \Gamma \boldsymbol{y} \boldsymbol{x} C_{\boldsymbol{x}\left(s^{\prime}\right)} \boldsymbol{x}\left(s^{\prime}\right) \Gamma \boldsymbol{x} \boldsymbol{y}$. Here we define the product of line contours as an oriented contour in which each line is connected at a common point. Also we used different symbols $C_{\boldsymbol{x} \boldsymbol{y}}$ and $\Gamma_{\boldsymbol{x} \boldsymbol{y}}$ for line contours, in order to distinguish a pair of lines $\Gamma \boldsymbol{x} \boldsymbol{y}$ introduced in the field redefinition (5) from the other lines $C_{\boldsymbol{x} y}$ which are segments of loops $C$ and $C^{\prime}$.

The part corresponding to the splitting of strings is given by

$$
\sum_{C} \operatorname{Tr}\left(T^{b} \Phi[\Gamma \boldsymbol{x} \boldsymbol{y}] T^{a} \Phi^{-1}[\Gamma \boldsymbol{x} \boldsymbol{y}]\right) \frac{\delta^{2} W(C)}{\delta A_{b}^{\mu}(\boldsymbol{x}) \delta A_{a}^{\mu}(\boldsymbol{y})} \frac{\delta}{\delta W(C)} .
$$

Similarly it can be rewritten in terms of Wilson loops as

$$
\begin{aligned}
& N \operatorname{Tr}\left(T^{b} \Phi\left[\Gamma_{\boldsymbol{x} \boldsymbol{y}}\right] T^{a} \Phi^{-1}\left[\Gamma_{\boldsymbol{x} \boldsymbol{y}}\right]\right) \frac{\delta^{2} W(C)}{\delta A_{b}^{\mu}(\boldsymbol{x}) \delta A_{a}^{\mu}(\boldsymbol{y})} \\
& =-\int_{0}^{2 \pi} d s\left[\int_{0}^{s} d s^{\prime} \operatorname{Tr}\left\{\left(P e^{i \int_{0}^{s^{\prime}} d s^{\prime \prime} \dot{\boldsymbol{x}}\left(s^{\prime \prime}\right) \cdot \boldsymbol{A}\left(\boldsymbol{x}\left(s^{\prime \prime}\right)\right)}\right) \Phi[\Gamma \boldsymbol{x} \boldsymbol{y}]\left(P e^{i \int_{s}^{2 \pi} d s^{\prime \prime} \dot{\boldsymbol{x}}\left(s^{\prime \prime}\right) \cdot \boldsymbol{A}\left(\boldsymbol{x}\left(s^{\prime \prime}\right)\right)}\right)\right\}\right. \\
& \times \operatorname{Tr}\left\{\left(P e^{i \int_{s^{\prime}}^{s} d s^{\prime \prime} \dot{\boldsymbol{x}}\left(s^{\prime \prime}\right) \cdot \boldsymbol{A}\left(\boldsymbol{x}\left(s^{\prime \prime}\right)\right)}\right) \Phi^{-1}[\Gamma \boldsymbol{x} \boldsymbol{y}]\right\}
\end{aligned}
$$

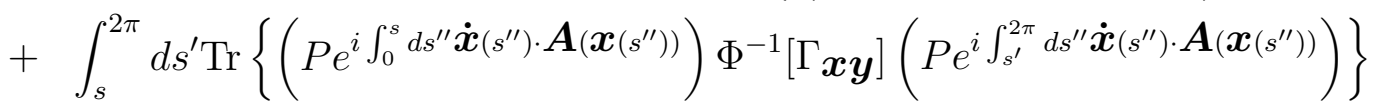

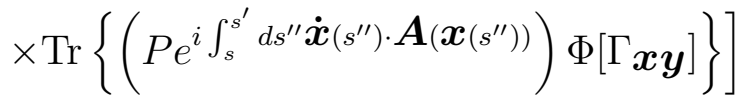

$$
\begin{aligned}
& \times\left(\dot{\boldsymbol{x}}(s) \cdot \dot{\boldsymbol{x}}\left(s^{\prime}\right)\right) \delta^{D}(\boldsymbol{x}(s)-\boldsymbol{y}) \delta^{D}\left(\boldsymbol{x}\left(s^{\prime}\right)-\boldsymbol{x}\right) \\
& =-N^{2} \int_{0}^{2 \pi} d s \int_{0}^{2 \pi} d s^{\prime}\left(\dot{\boldsymbol{x}}(s) \cdot \dot{\boldsymbol{x}}\left(s^{\prime}\right)\right) \delta^{D}(\boldsymbol{x}(s)-\boldsymbol{y}) \delta^{D}\left(\boldsymbol{x}\left(s^{\prime}\right)-\boldsymbol{x}\right) W\left(C_{B}\right) W\left(C_{\bar{B}}\right),
\end{aligned}
$$

where a loop $C$ is broken into two loops $C_{B}$ and $C_{\bar{B}}$, and they are respectively given by $C_{B}=\Gamma \boldsymbol{x} \boldsymbol{y} C \boldsymbol{x}_{(s)} \boldsymbol{x}\left(s^{\prime}\right)$ and $C_{\bar{B}}=C_{\boldsymbol{x}\left(s^{\prime}\right)} \boldsymbol{x}(s) \Gamma \boldsymbol{y} \boldsymbol{x}$.

Finally the second term in eq.A.1) corresponds to the kinetic term of the string field,

$$
\sum_{C} \operatorname{Tr}\left(T^{b} \Phi[\Gamma \boldsymbol{x} \boldsymbol{y}] \frac{1}{g_{b}^{2}} D^{\nu} F_{\nu \mu}(\boldsymbol{y}) \Phi^{-1}[\Gamma \boldsymbol{x} \boldsymbol{y}]\right) \frac{\delta W(C)}{\delta A_{b}^{\mu}(\boldsymbol{x})} \frac{\delta}{\delta W(C)}
$$




$$
\begin{aligned}
& =\sum_{C} \frac{i}{g_{b}^{2} N} \int_{0}^{2 \pi} d s \dot{x}^{\mu}(s) \delta^{D}(\boldsymbol{x}(s)-\boldsymbol{x}) \\
\times & \operatorname{Tr}\left\{\left(P e^{i \int_{0}^{s} d s^{\prime} \dot{\boldsymbol{x}}\left(s^{\prime}\right) \cdot \boldsymbol{A}\left(\boldsymbol{x}\left(s^{\prime}\right)\right)}\right) \Phi[\Gamma \boldsymbol{x} \boldsymbol{y}] D^{\nu} F_{\nu \mu}(\boldsymbol{y}) \Phi^{-1}[\Gamma \boldsymbol{x} \boldsymbol{y}]\left(P e^{i \int_{s}^{2 \pi} d s^{\prime} \dot{\boldsymbol{x}}\left(s^{\prime}\right) \cdot \boldsymbol{A}\left(\boldsymbol{x}\left(s^{\prime}\right)\right)}\right)\right\} \frac{\delta}{\delta W(C)} \\
& =\left.\frac{1}{g_{b}^{2}} \int_{0}^{2 \pi} d s \delta^{D}(\boldsymbol{x}(s)-\boldsymbol{x}) \frac{\delta^{2} W(C)}{\delta x\left(s_{0}\right)^{2}}\right|_{\boldsymbol{x}\left(s_{0}\right)=\boldsymbol{y}} \frac{\delta}{\delta W(C)},
\end{aligned}
$$

where we introduced a local derivative of the loop space [17],

$$
\frac{\delta^{2}}{\delta x(s)^{2}}=\lim _{\epsilon \rightarrow 0} \int_{-\epsilon}^{\epsilon} d t \frac{\delta^{2}}{\delta x^{\mu}(s+t / 2) \delta x_{\mu}(s-t / 2)} .
$$

\section{References}

[1] N. Seiberg, "Why Is The Matrix Model Correct?," Phys. Rev. Lett. 79 (1997) 3577, hep-th/9710009;

A. Sen, "D0-Branes On $T^{n}$ And Matrix Theory," Adv. Theor. Math. Phys. 2 (1998) 51, hep-th/9709220.

[2] T. Banks, W. Fishler, S. Shenker and L. Susskind, "M-Theory As A Matrix Model: A Conjecture," Phys. Rev. D55 (1997) 5112, hep-th/9610043;

L. Susskind, "Another Conjecture About M(atrix) Theory," hep-th/9704080.

[3] J. Maldacena, "The Large N Limit Of Superconformal Field Theories And Supergravity," Adv. Theor. Math. Phys. 2 (1998) 231, hep-th/9711200;

See also for a review, O. Aharony, S. S. Gubser, J. Maldacena, H. Ooguri and Y. Oz, "Large N Field Theories, String Theory And Gravity," hep-th/9905111.

[4] N. Itzhaki, J. Maldacena, J. Sonnenschein and S. Yankielowicz, "Supergravity And The Large $N$ Limit Of Theories With Sixteen Supercharges," Phys. Rev. D58 (1998) 46004, hep-th/9802042.

[5] J. Polchinski, "Dirichlet-Branes And Ramond-Ramond Charges," Phys. Rev. Lett. 75 (1995) 4724, hep-th/9510017; See also for a review, "TASI Lectures On Dbranes," hep-th/9611050.

[6] S. S. Gubser, I. R. Klebanov and A. M. Polyakov, "Gauge Theory Correlators From Non-critical String Theory," Phys. Lett. B428 (1998) 105, hep-th/9802109.

[7] E. Witten, "Anti-de Sitter Space And Holography," Adv. Theor. Math. Phys. 2 (1998) 253, hep-th/9802150.

[8] L. Susskind and E. Witten, "The Holographic Bound in Anti-de Sitter Space," hepth/9805114. 
[9] A. W. Peet and J. Polchinski, "UV/IR Relations In AdS Dynamics," Phys. Rev. D59 (1999) 065011, hep-th/9809022.

[10] K. G. Wilson and J. Kogut, "The Renormalization Group And The $\epsilon$ Expansion," Phys. Rep. 12C (1974) 75.

[11] B. Sakita, "Field Theory Of Strings As A Collective Field Theory Of U(N) Gauge Fields," Phys. Rev. D21 (1980) 1067;

A. Jevicki and B. Sakita, "The Quantum Collective Field Method And Its Application To The Planar Limit," Nucl. Phys. B165 (1980) 511.

[12] E. T. Akhmedov, "A Remark On The AdS/CFT Correspondence And The Renormalization Group Flow," Phys. Lett. B442 (1998) 152, hep-th/9806217;

E. Alvarez and C. Gomez, "Geometric Holography, The Renormalization Group And The c-Theorem," Nucl. Phys. B541 (1999) 441, hep-th/9807226;

M. Porrati and A. Starinets, "RG Fixed Points In Supergravity Duals of 4-d Field Theory And Asymptotically AdS Spaces," Phys. Lett. B454 (1999) 77, hepth/9903085;

V. Balasubramanian and P. Kraus, "Spacetime And The Holographic Renormalization Group," hep-th/9903190;

H. Verlinde, "Holography and Compactification," hep-th/990618;

O. Andreev, "Probing $\mathrm{AdS}_{3} / \mathrm{CFT}$ Correspondence Via World-Sheet Methods And 2d Gravity Like Scaling Arguments," hep-th/9909222.

[13] S.-J. Rey, private communication.

[14] T. R. Morris, "A Manifestly Gauge Invariant Exact Renormalization Group," hepth/9810104; "A Gauge Invariant Exact Renormalization Group I," hep-th/9910058.

[15] J. Polchinski, "Renormalization And Effective Lagrangian," Nucl. Phys. B231 (1984) 269.

[16] Y. M. Makeenko and A. A. Migdal, "Exact Equation For The Loop Average In Multicolor QCD," Phys. Lett. 88B (1979) 135; "Quantum Chromodynamics As Dynamics Of Loops," Nucl. Phys. B188 (1981) 269.

[17] A. M. Polyakov, "Gauge Fields and Strings," (1987), Harwood Academic Publishers.

[18] M. B. Halpern and Y. Makeenko, "Continuum Regularized Loop Space Equation," Phys. Lett. B218 (1989) 230; See also for a review, M. B. Halpern, "Universal Continuum Regularization Of Quantum Field Theory," Prog. Theor. Phys. Suppl. 111 (1993) 163. 
[19] J. M. Maldacena, "Wilson Loops In Large $N$ Field Theories," Phys. Rev. Lett. 80 (1998) 4859, hep-th/9803002;

S.-J. Rey and J. Yee, "Macroscopic Strings As Heavy Quarks Of Large $N$ Gauge Theory And Anti-de Sitter Supergravity," hep-th/9803001.

[20] D. Berenstein, R. Corrado, W. Fischler and J. Maldacena, "The Operator Product Expansion For Wilson Loops And Surfaces In The Large $N$ Limit," Phys. Rev. D59 (1999) 105023, hep-th/9809188.

[21] N. Drukker, D. J. Gross and H. Ooguri, "Wilson Loops And Minimal Surfaces," hep-th/9904191;

N. Drukker, "A New Type Of Loop Equations," hep-th/9908113.

[22] V. Periwal, "String Field Theory Hamiltonians From Yang-Mills Theories," hepth/9906052; "A Toy Model Of Polyakov Duality," hep-th/9908203;

G. Lifschytz and V. Periwal, "Dynamical Truncation Of The String Spectrum At Finite $N$," hep-th/9909152.

[23] N. Ishibashi and H. Kawai, "String Field Theory Of Noncritical Strings," Phys. Lett. B314 (1993) 190, hep-th/9307045;

See also for a review, M. Ikehara, N. Ishibashi, H. Kawai, T. Mogami, R. Nakayama and N. Sasakura, "A Note On String Field Theory In The Temporal Gauge," Prog. Theor. Phys. Suppl. 118 (1995) 241, hep-th/9409101.

[24] A. Jevicki and J. Rodrigues, "Loop Space Hamiltonians And Field Theory Of Noncritical Strings," Nucl. Phys. B421 (1994) 278, hep-th/9312118.

[25] G. Marchesini, "A Comment On The Stochastic Quantization: The Loop Equation Of The Gauge Theory As The Equilibrium Condition," Nucl. Phys. B191 (1981) 214.

[26] A. M. Polyakov, "String Theory And Quark Confinement," Nucl. Phys. Proc. Suppl. 68 (1998) 1, hep-th/9711002. 